\title{
THESES
}

\section{IDENTIFICATION OF THE MOTOR CORTEX DURING INTRACRANIAL SURGERY FOR BRAIN LESIONS UNDER GENERAL ANAESTHESIA (ABSTRACT)*. THESIS. RECIFE, 2004.}

\author{
PAULO THADEU BRAINER LIMA **
}

In the present descriptive observational clinical study an analysis was made of the following: the indications for surgery, the surgical findings and the detail of mapping, with direct electrical stimulation of the cortex and white matter under general anesthesia during surgery to brain tumor related to the central lobe.

All patients met the criteria for surgical treatment and the Karnofsky index was greater than 70. For stimulation purposes, a bipolar stimulator was used, which initially identified the area of the cortex responsible for the primary control of the hand. Successive stimulations were performed in the subcortical plane to locate the motor pathways during the resection of the tumor. Stereotaxic marking was used in 12 (28.6\%) patients. Age ranged from 8 to 70 years (mean, 38.43\%). The commonest complaint was epilepsy (69\%). The neurological examination was normal in $35.7 \%$. Tumors were related to the frontal lobe in $73.8 \%$. Astrocytomas were the most frequent histological type, with 16 cases accounting for $41.1 \%$ of the total, $21.4 \%$ being low grade tumor (grade II), with 6 oligodendroglioma (14,3\%), 2 glioblastoma (4.8\%), 5 metastases $(11.9 \%), 4$ cysticercoses $(9.5 \%), 3$ meningiomas $(7.1 \%), 3$ cavernomas $(7.1 \%), 1$ abscess $(2.4 \%), 1$ arteriovenous malformation (2.4\%) and 1 tuberculoma (2.4\%).

Patients were grouped on the basis of the histology as follows: $24(57.2 \%)$ patients with primary brain tumor (Group A) and $18(42.8 \%)$ with the secondary brain tumor (Group B). All patients were investigated using MRI. Contrast enhancement occurred in all Group B patients and in 19 (79.2\%) patients in Group $A$. The stimulus on the cortex was from 4 to $18 \mathrm{~mA}$, with a mean of $10.33 \mathrm{~mA}$, no differences being found between groups $A$ and $B$ ( $p$
$=0.9366 ; \mathrm{T}=-0.08)$. The mean value of the intensity of the stimulus was greater among patients over 45 years old $(p=0.5819)$ and among those who presented motor deficit prior to surgery $(p=0.0425)$, edema in MRI $(p=0.0468)$ or during anesthesia with continuous propofol (13.26 mA), differently from what occurred with intermittent administration (8.0 $m A)$; t-Student, $P<0.001$ ).

Surgical resection was complete in 12 patients $(50.0 \%)$ in Group A and in 17 (94.4\%) in Group B. Among the 25 patients with no or mild motor deficit preoperatively, 11 were classified as having a moderate or severe deficit in the immediate postoperative period (significant worsening; $\chi^{2}=8.3333$ $\mathrm{p}=0.0039)$. Of the $27(100 \%)$ patients considered as having a moderate or severe deficit in the immediate postoperative examination, $20(74.1 \%)$ progressed to a mild degree or absence of deficit (significant improvement $\chi^{2}=20.0 p<0.001$ ). Surgical complications occurred in $5(11.9 \%)$ patients, of whom underwent a second operation. The Karnofsky index rose within sucessive follow-up evaluations. For each type of tumor a comparison of initial and final indices showed a significant improvement $(T=6.23 p<0.001)$. The follow up time of the patients was ranging from 3 to 45 months (mean of 11 months) with a significant recovery from the motor deficit existing prior to surgery $(p<$ 0.001).

The functional mapping of the central lobe was influenced by severe motor deficit, edema on MRI and proprofol's anesthesia. The motor deficit following surgery was transient and the patient's functional status showed a significant improvement.

KEY WORDS: functional surgery, motor cortex, central cortex, stimulation, brain tumor, mapping.

*Identificação da area motora, sob anestesia geral, durante cirurgia para retirada de tumor intracraniano (Resumo). Tese de Doutorado, Universidade Federal de Pernambuco (Área: Cirurgia). Orientador: Carlos Teixeira Brandt. 